\title{
Simulation study of magnetic fields generated by the electromagnetic filamentation instability
}

\author{
K.-I. Nishikawa ${ }^{*}$, E. Ramirez-Ruiz ${ }^{\dagger}$, P. Hardee ${ }^{* *}$, C. B. Hededal ${ }^{\ddagger}$, Y. Mizuno* and \\ G. J. Fishman ${ }^{\S}$ \\ *National Space Science and Technology Center, Huntsville, AL 35805 \\ ${ }^{\dagger}$ Institute for Advanced Study, Einstein Drive, Princeton, NJ 08540 \\ ** Department of Physics and Astronomy, The University of Alabama, Tuscaloosa, AL 35487 \\ ${ }^{\ddagger}$ Dark Cosmology Center, Niels Bohr Institute, Juliane Maries Vej 30 , \\ 2100 Copenhagen $\emptyset$, Denmark \\ $\S_{\text {NASAMMSFC, Huntsville, AL } 35805}$
}

\begin{abstract}
We have investigated the effects of plasma instabilities driven by rapid $e^{ \pm}$pair cascades, which arise in the environment of GRB sources as a result of back-scattering of a seed fraction of the original spectrum. The injection of $e^{ \pm}$pairs induces strong streaming motions in the ambient medium. One therefore expects the pair-enriched medium ahead of the forward shock to be strongly sheared on length scales comparable to the radiation front thickness. Using threedimensional particle-in-cell simulations, we show that plasma instabilities driven by these streaming $e^{ \pm}$pairs are responsible for the excitation of near-equipartition, turbulent magnetic fields. Our results reveal the importance of the electromagnetic filamentation instability in ensuring an effective coupling between $e^{ \pm}$pairs and ions, and may help explain the origin of large upstream fields in GRB shocks.
\end{abstract}

Keywords: Pair loading, Weibel instability, magnetic field generation

PACS: $98.70 . \mathrm{Rz}$ gamma-ray sources; gamma-ray bursts

\section{RPIC SIMULATIONS}

Particle-in-cell (PIC) simulations can shed light on the physical mechanism of particle acceleration that occurs in the complicated dynamics within relativistic shocks. Recent PIC simulations using injected relativistic electron-ion and electron-positron jets show that acceleration occurs within the downstream jet $[1,2,3]$. In general, these simulations have confirmed that relativistic jets excite the Weibel instability. The Weibel instability generates current filaments and associated magnetic fields [4], and accelerates electrons [1,2,3].

Three simulations were performed using an $85 \times 85 \times 640$ grid with a total of 380 million particles (27 particles/cell/species for the ambient plasma) and an electron skin depth, $\lambda_{\text {ce }}=c / \omega_{\mathrm{pe}}=9.6 \Delta$, where $\omega_{\mathrm{pe}}=\left(4 \pi e^{2} n_{\mathrm{e}} / m_{\mathrm{e}}\right)^{1 / 2}$ is the electron plasma frequency and $\Delta$ is the grid size. In all simulations jets are injected at $z=25 \Delta$ in the positive $z$ direction. In all simulations radiating boundary conditions were used on the planes at $z=0, z_{\max }$. Periodic boundary conditions were used on all other boundaries. The ambient and jet electron-positron plasma has mass ratio $m_{\mathrm{e}} / m_{\mathrm{p}} \equiv m_{\mathrm{e}^{-}} / m_{\mathrm{e}^{+}}=1$. The electron thermal velocity in the ambient plasma is $v_{\text {th }}=0.1 c$ where $c$ is the speed of light. The mass ration on ions in the ambient plasma is $m_{\mathrm{i}} / m_{\mathrm{e}}=20$

In this report we simulate three different initial jet electrons (positrons) distributions. The first case (A) is as same as one of the simulations in previous papers $[2,3]\left(\gamma V_{\|}=12.57 c\right)$. The second $(\mathrm{B})$ and third cases $(\mathrm{C})$ are a special case and corresponds to cold jet electrons and positrons created by photon annihilation with $2 c<\gamma V_{\|}<30 c$ [5]. For all cases the temperature of jet particles are very cold $(0.01 c$ in the rest frame). In this letter we concentrate on the simulation with the broad $2<\gamma V_{\|}<30$ Lorentz factor distribution. The first (A) and second (B) cases are injected into electron-ion ambient plasma, the third $(C)$ case is injected in ambient pair plasma. The average jet velocity for the broad pair loading is nearly $\gamma V_{\|}=12.5 \mathrm{c}$.

Figure 1 shows snapshots for three cases (top: A, middle: B, and bottom: C). The Weibel instability in the electronpositron jet injected at $z / \Delta=25$ is illustrated in 2D images in the $x-z$ plane $(y=43 \Delta)$ in the center of the jet at $t=59.8 / \omega_{\text {pe }}$. In the left column the colors indicate the $z$-component of the current density $\left(J_{z}\right)$ with $J_{z}, J_{\mathrm{x}}$ indicated by the arrows. Behind the jet front $(z / \Delta=600)$ the Weibel instability perturbs the electron density, leading to nonuniform currents and transverse magnetic fields $[2,3]$. The magnetic field energy density averaged in the $x-y$ plane along the $z$-direction. The total energy density $\left(0.5 *\left(B_{\mathrm{x}}^{2}+B_{\mathrm{y}}^{2}+B_{\mathrm{z}}^{2}\right)\right)$ is plotted by the red curves, the perpendicular energy 

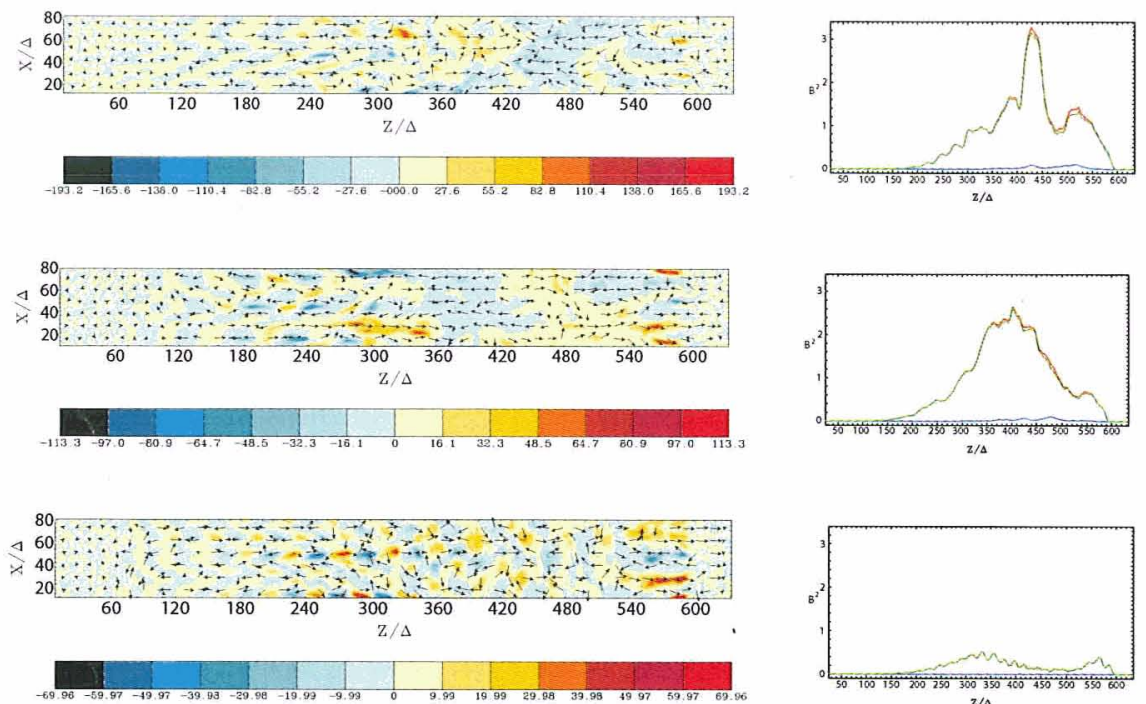

FIGURE 1. The snap shots of simulation with at $t=59.8 / \omega_{\mathrm{pe}}$ for three cases. In the left column the colors indicate the $Z$ component of the current density $\left(J_{\mathrm{Z}}\right)$ with $J_{\mathrm{Z}}, J_{\mathrm{x}}$ indicated by the arrows. In the right column the total energy density $\left(0.5 *\left(B_{\mathrm{x}}^{2}+\right.\right.$ $\left.\left.B_{\mathrm{y}}^{2}+B_{\mathrm{z}}^{2}\right)\right)$ is plotted by the red curves, the perpendicular energy density $\left(0.5 *\left(B_{\mathrm{x}}^{2}+B_{\mathrm{y}}^{2}\right)\right)$, by green, and the parallel energy density $\left(0.5 * B_{\mathrm{Z}}^{2}\right)$, by blue curves.

density $\left(0.5 *\left(B_{\mathrm{x}}^{2}+B_{\mathrm{y}}^{2}\right)\right)$, by green, and the parallel energy density $\left(0.5 * B_{\mathrm{z}}^{2}\right)$, by blue curves. The Weibel instability in the electron-positron jet injected at $z / \Delta=25$ is illustrated in 2D images in the $x-z$ plane $(y=43 \Delta)$ in the center of the jet at $t=59.8 / \omega_{\mathrm{pe}}$. In the left column the colors indicate the $z$-component of the current density $\left(J_{\mathrm{z}}\right)$ with $J_{\mathrm{z}}, J_{\mathrm{x}}$ indicated by the arrows. Behind the jet front $(z / \Delta=600)$ the Weibel instability perturbs the electron density, leading to nonuniform currents and transverse magnetic fields.

The comparison among three cases shows that the electron-ion ambient plasma enhances the generation of magnetic field due to the excitation of ion filamentation instability. The pair jet with broad distribution sustains the magnetic field strength due to the longer linear stage with jet particles with larger Lorenz factor. Further discussions will be reported separately [6].

\section{ACKNOWLEDGMENTS}

K.N. is partially supported by the National Science Foundation award AST-0506719, and the National Aeronautic and Space Administration award NASA-INTEG04-0000-0046 and HST-AR-10966.01-A. Y. M. is supported by an appointment of the NASA Postdoctoral Program at NASA Marshall Space Flight Center, administered by Oak Ridge Associated Universities through a contract with NASA. P. H. acknowledges partial support by National Space Science and Technology Center (NSSTC/NASA) cooperative agreement NCC8-256 and National Science Foundation (NSF) award AST-0506666 to the University of Alabama. The simulations have been performed on Columbia at NASA Advanced Supercomputing (NAS) and IBM p690 (Copper) at the National Center for Supercomputing Applications (NCSA) which is supported by the National Science Foundation.

\section{REFERENCES}

1. K.-I. Nishikawa, P. Hardee, G. Richardson, R. Preece, H. Sol, H., and G. J. Fishman, ApJ, 595, 555-563 (2003)

2. K.-I. Nishikawa, P. Hardee, G. Richardson, R. Preece, H. Sol, H., and G. J. Fishman, ApJ, 623, 927-937 (2005)

3. K.-I. Nishikawa, P. Hardee, C. B. Hededal, and G. J. Fishman, ApJ, 642, 1267-1274 (2006)

4. M. V. Medvedev, and A. Loeb, ApJ, 526, 697-706 (1999)

5. P. Meszaros, E. Ramirez-Ruiz, and M J. Rees, ApJ, 554, 660-666 (2001)

6. E. Ramirez-Ruiz, K.-I. Nishikawa, and C. B. Hededal, ApJL, submitted (2006) 